\title{
Correlation of the Neutrophil-to-Lymphocyte Ratio (NLR) and Platelet-to-Lymphocyte Ratio (PLR) with Contrast-Induced Nephropathy in Patients With Acute Coronary Syndrome Undergoing Percutaneous Coronary Interventions
}

Khurram Butt ${ }^{1}$, Jason D'Souza ${ }^{2}$, Cai Yuan ${ }^{3}$, Jayapriya Jayakumaran ${ }^{4}$, Michelle Nguyen ${ }^{5}$, Hamza I. Butt ${ }^{6}$, Khalid Abusaada ${ }^{5}$

1. Internal Medicine, AdventHealth Orlando, Orlando, USA 2. Cardiology, St. Luke's Health System, University of Missouri, Kansas City, USA 3. Oncology, University of Florida, Gainesville, USA 4. Internal Medicine, Ocala Regional Medical Center, University of Central Florida College of Medicine, Orlando, USA 5. Internal Medicine, Ocala Regional Medical Center, University of Central Florida College of Medicine, Ocala, USA 6. Epidemiology and Public Health, Mel and Enid Zuckerman College of Public Health, University of Arizona, Tucson, USA

Corresponding author: Hamza I. Butt, hamzabutt39@email.arizona.edu

\begin{abstract}
Introduction

Contrast-induced acute nephropathy (CIN) in patients undergoing percutaneous coronary intervention (PCI) in the setting of acute coronary syndromes (ACS) is associated with adverse outcomes, including longer hospitalization and short and long-term mortality. Neutrophil to lymphocyte ratio (NLR) and platelet to lymphocyte ratio (PLR) are inflammatory markers that have been validated separately in prior studies as a predictor of CIN in patients with ACS who undergo a left heart catheterization. Our study aims to further investigate the role of NLR and PLR together as markers for predicting CIN in patients with ACS.
\end{abstract}

Methods

A retrospective chart review was performed on a total of 1,577 patients aged 18 - 90 who presented with ACS and underwent PCI between January 2011 to December 2015 at the Florida Hospital Orlando. Cut-off values used for a high PLR and NLR were PLR $>128$ and NLR $>2.6$. CIN was defined as an increased serum creatinine level by $\geqslant 0.5 \mathrm{mg} / \mathrm{dL}$, or $\geqslant 25 \%$, over the baseline value within 72 hours after contrast agent administration. Patients with end-stage renal disease (ESRD) were excluded.

Results

Review began $11 / 11 / 2020$ Review ended 11/13/2020 Published 12/03/2020

\section{() Copyright 2020}

Butt et al. This is an open access article distributed under the terms of the Creative Commons Attribution License CC-BY 4.0., which permits unrestricted use, distribution, and reproduction in any medium, provided the original author and source are credited.
Of the 1,577 patients included in the study, 213 (13.51\%) patients had CIN. On multivariate logistic regression analysis, high NLR showed an independent association with an elevated risk of CIN (OR 2.03, 95\% CI: 1.403 - 3.176, P < 0.001). High PLR did not correlate with CIN (OR 0.831, 95\% CI: 0.569 - 1.214, P = $0.339)$.

Conclusion

Elevated NLR is an independent predictor of CIN in patients with acute myocardial infarction (AMI) and may be used to improve on current risk prediction models.

Categories: Cardiac/Thoracic/Vascular Surgery, Cardiology, Nephrology

Keywords: cardiac catheterization, nephropathy, percutaneous coronary intervention (pci), neutrophil to lymphocyte ratio (nlr), coronary artery disease (cad), contrast-induced nephropathy (cin), acute myocardial infarction (ami), acute kidney injury (aki), platelet to lymphocyte ratio (plr)

\section{Introduction}

Contrast-induced nephropathy (CIN) is the third leading cause of in-hospital acute kidney injury (AKI) [1] and is associated with significant in-hospital and long-term morbidity and mortality [2-3]. The pathogenesis of CIN is multifactorial and multiple mechanisms have been suggested; inflammation and prothrombotic milieu are known to play a key role. Hence, mediators reflecting inflammation and thrombosis, such as neutrophil to lymphocyte ratio (NLR) and platelet to lymphocyte ratio (PLR), are being studied as markers of CIN [4]. The interest in the association between NLR and PLR and the risk of cardiovascular events in patients undergoing angiography or percutaneous coronary intervention (PCI) is rapidly growing. Multiple studies have established an association of PLR and NLR with the risk of CIN in ST-elevation myocardial 
infarction (STEMI) and non-ST-elevation myocardial infarction (NSTEMI) patients [5-7]. However, the utility of these markers in combination with other factors in predicting CIN in acute myocardial infarction (AMI) has not been studied. In this study, we assessed the correlation between NLR and PLR and CIN in patients with AMI and to evaluate the effect of these scores on the performance of the Mehran score to predict the risk of CIN in this population [8].

\section{Materials And Methods}

This was a retrospective analysis of data collected as part of the Acute Coronary Treatment and Intervention Outcomes Network (ACTION) Registry ${ }^{\circledR}$ (now called the Chest Pain-MI Registry ${ }^{\mathrm{TM}}$ ) [9] at a tertiary care center between January 1, 2011 and December 31, 2015. Data in the Registry is collected prospectively for patients who present within 24 hours of the onset of an ischemic event with a primary diagnosis of myocardial infarction (STEMI or NSTEMI). In addition, the research team conducted a chart review of all included patients to obtain the following data that is not collected as part of the Chest Pain-MI Registry: absolute neutrophil and lymphocyte counts, platelet count, the highest creatinine recorded within 72 hours of left heart catheterization and PCI, contrast volume, and the use of intra-aortic balloon pump (IABP). The study was approved by the Advent Health Institutional Review Board (approval 868065). Patients were excluded if they did not undergo a PCI, were receiving long-term renal replacement therapy prior to admission, or if no creatinine level was obtained within 72 hours of the PCI.

NLR and PLR were defined as the ratio of the absolute neutrophil count and the absolute platelet count, respectively, to the absolute lymphocyte count. CIN was defined as a rise in the serum creatinine level by $\geqslant$ $0.5 \mathrm{mg} / \mathrm{dL}$ or $\geqslant 25 \%$ over the baseline value within 72 hours after the contrast agent was administered. Cardiogenic shock is defined as having one or more of the following conditions: (a) a sustained decline of $>30$ minutes of systolic blood pressure to $<90 \mathrm{~mm} \mathrm{Hg}$ or a cardiac index $<2.2 \mathrm{~L} / \mathrm{min} / \mathrm{m}^{2}$; (b) requirement for parenteral inotropic/vasopressor agents or mechanical support to maintain a systolic blood pressure $\geqslant 90 \mathrm{~mm} \mathrm{Hg}$ or cardiac index $\geqslant 2.2 \mathrm{~L} / \mathrm{min} / \mathrm{m}^{2}$. Tachycardia was defined as a heart rate on admission of $>100$ beats per minute.

Association of AKI and NLR or PLR was evaluated using the Student t-test. NLR and PLR were categorized using cut-off values established by Cho et al. [10]. An NLR level over 2.6 and a PLR level over 128 were considered high. Baseline characteristics of patients with high NLR and PLR levels were compared to those with low NLR and PLR levels, respectively, in a bivariate analysis using the Student's t-test for continuous variables and the Chi-square test for categorical variables. A two-sided $\mathrm{p}$-value of $\leqslant 0.05$ was considered statistically significant.

A multivariate logistic regression model was built with CIN as the outcome and the following variables as covariates: PLR, NLR, left ventricular ejection fraction, presence of acute congestive heart failure (CHF) at time of admission, presence of shock at the time of admission, cardiac arrest at the time of admission, diabetes, hypertension, age, gender, glomerular filtration rate (GFR), anemia, tachycardia, and the use of IABP.

Finally, we evaluated the contribution of NLR to the prediction of CIN in patients with AMI through comparison of the area under receiver operating characteristic curve (AUROC) and calibration between a Mehran score model and a Mehran score model, plus categorized NLR. The Mehran score was calculated based on the factors reported by Mehran et al. [8]. A two-sided p-value of $\leqslant 0.05$ was considered statistically significant for all comparisons. The data analysis was performed using Stata, version 13.0 (StataCorp LLC, College Station, TX, USA).

\section{Results}

A total of 1,577 patients were included in the analysis. Two hundred and thirteen (13.51\%) patients developed CIN. Patients with CIN had a higher NLR (6.9 vs 4.5, $\mathrm{p}=0.003$ ) and a higher PLR (164 vs 133, $\mathrm{p}<$ $0.001)$. High NLR was associated with CIN $(p<0.001)$, STEMI patients $(p=0.008)$, anemia $(p<0.001)$, CHF admission $(p=0.009)$, cardiac arrest on admission $(p=0.026)$, diabetes mellitus $(p=0.002)$, hypertension ( $p$ $=0.038)$, reduced left ventricular ejection fraction (LVEF) $(p<0.001)$, and tachycardia $(p<0.001)$ (Table 1). High PLR was associated with CIN $(p=0.002)$, male gender $(p=0.025)$, STEMI $(p=0.001)$, anemia $(p<$ $0.001)$, CHF on admission $(p=0.023)$, cardiac arrest on admission $(p=0.011)$, and hypertension $(p=0.025)$ (Table 2). 


\section{Cureus}

\begin{tabular}{|c|c|c|c|}
\hline Parameter & NLR $\leq \mathbf{2 . 8}$ & NLR $>2.8$ & p-value \\
\hline Age (years), mean $\pm S D$ & $61.10 \pm 0.47$ & $63.98 \pm 0.43$ & 1.000 \\
\hline CIN, n (\%) & $58(8.45 \%)$ & $155(16.95 \%)$ & $<0.001$ \\
\hline Male gender, $\mathrm{n}(\%)$ & $482(70.26 \%)$ & $626(70.26 \%)$ & 0.999 \\
\hline STEMI, n (\%) & $381(55.55 \%)$ & $435(48.82 \%)$ & 0.008 \\
\hline Anemia, n (\%) & $106(15.45 \%)$ & $202(22.67 \%)$ & $<0.001$ \\
\hline CHF at admission, $\mathrm{n}(\%)$ & $31(4.52 \%)$ & $69(7.74 \%)$ & 0.009 \\
\hline Shock admission, n(\%) & $5(0.73 \%)$ & $11(1.23 \%)$ & 0.320 \\
\hline Cardiac arrest at admission, $\mathrm{n}(\%)$ & $39(5.69 \%)$ & $30(3.37 \%)$ & 0.026 \\
\hline DM, n (\%) & $210(30.61 \%)$ & 338 (37.93\%) & 0.002 \\
\hline HTN, n (\%) & $492(71.72 \%)$ & $680(76.32 \%)$ & 0.038 \\
\hline IABP, n (\%) & $17(2.48 \%)$ & $35(3.93 \%)$ & 0.110 \\
\hline $\mathrm{GFR}(\mathrm{ml} / \mathrm{min})$, mean $\pm \mathrm{SD}$ & $76.09 \pm 0.87$ & $74.11 \pm 0.85$ & 0.055 \\
\hline LVEF, mean \pm SD & $50.94 \pm 0.42$ & $48.14 \pm 0.41$ & $<0.001$ \\
\hline Tachycardia, n (\%) & $107(15.60 \%)$ & $207(23.23 \%)$ & $<0.001$ \\
\hline Mortality, n (\%) & $20(2.92 \%)$ & $32(3.59 \%)$ & 0.456 \\
\hline
\end{tabular}

TABLE 1: Bivariate Analysis of Demographic and Clinical Parameters with Neutrophil to Lymphocyte Ratio (NLR) Category

CHF: congestive heart failure; CIN: contrast-induced acute kidney; DM: diabetes mellitus; HTN: hypertension; GFR: glomerular filtration rate; IABP: intra-aortic balloon pump; LVEF: left ventricular ejection fraction; SD: standard deviation; STEMI: ST-elevation myocardial infarction 


\section{Cureus}

\begin{tabular}{|c|c|c|c|}
\hline Variables & PLR $\leq 128$ & PLR > 128 & p-value \\
\hline Age (years), mean \pm SD & $61.42 \pm 0.40$ & $64.73 \pm 0.52$ & $<0.001$ \\
\hline CIN, n (\%) & $109(11.19 \%)$ & $104(16.53 \%)$ & 0.002 \\
\hline Gender, male, n (\%) & 693 (72.34\%) & $415(67.04 \%)$ & 0.025 \\
\hline STEMI, n (\%) & 527 (55.01\%) & $289(46.69 \%)$ & 0.001 \\
\hline Anemia, $n(\%)$ & $148(15.45 \%)$ & $160(25.85 \%)$ & $<0.001$ \\
\hline CHF at admission, $n(\%)$ & $50(5.22 \%)$ & 50 (8.08\%) & 0.023 \\
\hline Shock at admission, n (\%) & $11(1.15 \%)$ & $5(0.81 \%)$ & 0.510 \\
\hline Cardiac arrest at admission, $\mathrm{n}(\%)$ & $52(5.43 \%)$ & $17(2.75 \%)$ & 0.011 \\
\hline DM, n (\%) & $321(33.51 \%)$ & $227(36.67 \%)$ & 0.197 \\
\hline HTN, n (\%) & 693 (72.34\%) & $479(77.38 \%)$ & 0.025 \\
\hline Use of IABP, $n(\%)$ & 29 (3.03\%) & 23 (3.72\%) & 0.455 \\
\hline GFR $(\mathrm{ml} / \mathrm{min})$, mean $\pm \mathrm{SD}$ & $75.50 \pm 0.75$ & $74.18 \pm 1.05$ & 0.146 \\
\hline LVEF, mean $\pm S D$ & $49.65 \pm 0.38$ & $48.94 \pm 0.48$ & 0.121 \\
\hline Tachycardia, n (\%) & $164(17.12 \%)$ & $150(24.23 \%)$ & 0.001 \\
\hline Mortality, $\mathrm{n}(\%)$ & $32(3.34 \%)$ & $20(3.23 \%)$ & 0.906 \\
\hline
\end{tabular}

\section{TABLE 2: Demographic and Clinical Parameters by Platelet to Lymphocyte Ratio (PLR) Category}

CHF: congestive heart failure; CIN: contrast-induced acute kidney; DM: diabetes mellitus; HTN: hypertension; GFR: glomerular filtration rate; IABP: intra-aortic balloon pump; LVEF: left ventricular ejection fraction; SD: standard deviation; STEMI: ST-elevation myocardial infarction

Multivariable logistic regression showed that after adjusted for other covariates, high NLR remained significantly associated with increased risk of CIN (OR 2.03, 95\% CI 1.403-3.176, P<0.001) whilst high PLR was not (OR $0.831,95 \% \mathrm{CI} 0.569-1.214, \mathrm{P}=0.339$ ). Other factors significantly associated with increased risk of CIN were age, STEMI, shock on admission, cardiac arrest on admission, tachycardia, diabetes mellitus, hypertension, use of IABP, and anemia (Table 3). NLR level $>2.6$ had a sensitivity of $72 \%$, specificity of $46 \%$, positive predictive value of $17 \%$ and negative predictive value of $92 \%$. 


\section{Cureus}

\begin{tabular}{|c|c|c|c|}
\hline Variables & Odds ratio & 95\% Confidence Interval & p-value \\
\hline STEMI & 1.432 & $1.027-1.998$ & 0.034 \\
\hline GFR & 1.007 & $1.000-1.012$ & 0.056 \\
\hline LVEF & 0.994 & $0.981-1.007$ & 0.363 \\
\hline Anemia & 1.865 & $1.302-2.674$ & 0.001 \\
\hline NLR > 2.6 & 2.032 & $1.363-3.028$ & $<0.001$ \\
\hline PLR $>128$ & 0.858 & $0.590-1.247$ & 0.422 \\
\hline CHF admission & 1.652 & $0.971-2.809$ & 0.064 \\
\hline Shock at admission & 3.574 & $1.042-12.27$ & 0.043 \\
\hline Cardiac arrest at admission & 2.072 & $1.030-4.166$ & 0.041 \\
\hline DM & 1.688 & $1.385-2.706$ & 0.002 \\
\hline HTN & 2.149 & $1.320-3.496$ & 0.002 \\
\hline Age & 1.018 & $1.005-1.032$ & 0.007 \\
\hline Tachycardia & 1.721 & $1.200-2.468$ & 0.003 \\
\hline Use of IABP & 2.851 & $1.458-5.576$ & 0.002 \\
\hline
\end{tabular}

\section{TABLE 3: Multivariable Logistic Regression Model for Prediction of Contrast-induced}

\section{Nephropathy}

CHF: congestive heart failure; CIN: contrast-induced acute kidney; DM: diabetes mellitus; HTN: hypertension; GFR: glomerular filtration rate; IABP: intra-aortic balloon pump; LVEF: left ventricular ejection fraction; NLR: neutrophil to lymphocyte ratio; PLR: platelet to lymphocyte ratio; STEMI: STelevation myocardial infarction

To evaluate the utility of NLR in the prediction of CIN, we incorporated the categorized NLR into the Mehran score model (Table 4). All factors included in the Mehran score were significant predictors of CIN, except contrast volume. When NLR was added to the model, it was an independent significant predictor of CIN (OR 1.80, $\mathrm{p}<0.001$ ). The new model with the addition of NLR had better discrimination ability with AUROC ( 0.73 vs $0.71, \mathrm{p}=0.015)$.

\begin{tabular}{|c|c|c|c|c|}
\hline Risk Factors & Mehran model OR (95\% Cl) & p-value & Mehran with NLR OR (95\% CI) & p-value \\
\hline Hypotension & $5.42(1.69-17.37)$ & 0.004 & $5.34(1.66-17.13)$ & 0.005 \\
\hline Use of IABP & $4.53(2.40-8.55)$ & $<0.001$ & $4.43(2.34-8.39)$ & $<0.001$ \\
\hline Age $>75$ years & $1.82(1.28-2.60)$ & 0.001 & $1.75(1.22-2.50)$ & 0.002 \\
\hline Contrast & $1.11(0.93-1.32)$ & 0.220 & $1.11(0.93-1.32)$ & 0.247 \\
\hline DM & $1.86(1.35-2.55)$ & $<0.001$ & $1.82(1.33-2.51)$ & $<0.001$ \\
\hline $\mathrm{CHF}$ & $2.13(1.30-3.48)$ & 0.003 & 2.05 (1.25 - 3.37) & 0.005 \\
\hline CKD & $1.79(1.13-2.86)$ & 0.014 & $1.69(1.06-2.70)$ & 0.028 \\
\hline Anemia & $1.89(1.33-2.69)$ & $<0.001$ & $1.83(1.28-2.60)$ & 0.001 \\
\hline NLR $>2.6$ & & & $1.81(1.28-2.54)$ & 0.001 \\
\hline
\end{tabular}

TABLE 4: Mehran Model With and Without High Neutrophil to Lymphocyte Ratio (NLR)

CHF: congestive heart failure; Cl: confidence interval; CKD: chronic kidney disease; DM: diabetes mellitus; IABP: intra-aortic balloon pump; OR: odds ratio 


\section{Discussion}

In this study, NLR was independently associated with CIN and improved the performance of the Mehran score in predicting CIN in patients with AMI. PLR was not independently associated with CIN in our study.

The etiology of CIN in the setting of AMI is complex. Besides the toxicity of contrast agents, abnormal hemodynamic changes associated with AMI may result in decreased renal blood flow, increased thrombogenic state, activation of inflammatory and neurohormonal cascades, and increased oxidative stress. Studies have demonstrated a strong association between inflammation and renal injury. It has been shown that infiltration of damaged tissue by inflammatory cells leads to worsening of renal injury [11].

The NLR has been a special marker of interest due to its role as a prognostic factor for coronary artery disease (CAD), hypertension, chronic kidney disease (CKD), diabetes, heart failure, cerebrovascular disease, peripheral arterial disease, and malignancy [12]. The plausible pathophysiological mechanism for this relationship is the role of neutrophils in mediating the inflammatory response to acute myocardial injury, causing further tissue damage [13]. The release of reactive oxygen species, myeloperoxidase, and proteolytic enzymes facilitate plaque rupture [14-15]. Lymphocytes play a major role in regulating the immune system [16] and inflammation enhances lymphocytic apoptosis [17].

In several prior studies, a high NLR was associated with increased mortality in patients with STEMI [5, 18]. To date, however, only three studies have explored the association of NLR with CIN in patients undergoing PCI for AMI [19-21]. The prospective study by Kurtul et al. specifically studied the subgroup of AMI patients that presented with NSTEMI [19]. In their study, they demonstrated that a high NLR (> 3.46) had a 73\% specificity and a 70\% sensitivity for CIN (area under the curve: 0.787 ), similar to our findings of a ROC of 0.73 when we added NLR to the Mehran score. On the other hand, Kaya et al. studied the role of NLR with CIN in the STEMI population undergoing PCI [20]. They demonstrated that a high NLR (> 6.35) had a sensitivity and specificity of $75 \%$ and $65 \%$, respectively, with an AUC of 0.763 . Similar findings were noted by Yuan et al. in their study of STEMI patients wherein an NLR > 5.71 had a sensitivity and specificity of $74 \%$ and 55\%, respectively, with an AUC of 0.708 [21].

Although the association between NLR and CIN has been shown before, our study is novel in its attempt to explore the practical utility of this marker as a predictor of CIN and its interaction with other established risk factors. The Mehran score is one of the well-studied risk score systems to predict CIN and has been validated in patients with AMI [22]. Our study shows that the addition of NLR to Mehran score improved the discriminatory capacity of the score. Thus, NLR could be utilized to predict CIN in AMI patients. Improvement in the prediction model would allow earlier implementation of cardioprotective measures in high-risk patients.

In addition, this is the first study (to the best of our knowledge) to report the utility of NLR as an independent predictor of CIN in a combined group of NSTEMI and STEMI patients in the American population. Based on our results, NLR independently predicted CIN with modest accuracy using a cutoff value of 2.6. NLR with this cutoff has a high negative predictive value that could be clinically useful. Other factors significantly associated with CIN were age, anemia, diabetes mellitus, hypertension, LVEF, CHF admission, tachycardia, use of IABP, and cardiac arrest.

Previous studies have linked a high PLR ratio to increased incidence of CIN in AMI [7]. To date, there are only three studies that have evaluated the role of PLR and its association with CIN in AMI patients who underwent PCI. However, two of these were studied in the STEMI subgroup of patients. Sun et al. noted that a PLR cutoff of $>127.5$ was associated with a sensitivity and specificity of $76.8 \%$ and $69.2 \%$ respectively [23]. On the other hand, Velibey et al. demonstrated that a PLR cutoff of $>177.5$ had a sensitivity and specificity of $60 \%$ and $72 \%$, respectively; however, when they excluded patients with anemia, PLR was not found to have a significant association with CIN [24]. This could be explained by the fact that iron deficiency anemia is associated with thrombocytosis and thus a higher PLR.

In our study, PLR was not found to have an independent correlation with CIN in AMI patients who underwent PCI. We used a cut-off value of 128 as high PLR as per the study by Cho et al. [10]; however, even when a cutoff value of PLR > 177 (as in the study by Velibey et al.) was used, it remained nonsignificant on the multivariate logistic regression model [24]. We are unable to explain this discrepancy in findings. This potentially could be due to differences in the population (American in our study vs Turkish) or different factors included in the regression model (for example, Velibey et al. did not adjust for CHF on admission or use of IABP) [24]. Further prospective studies are needed to validate the role of PLR in the prediction of CIN in AMI patients.

\section{Limitations}

Firstly, the inherent limitation of the study is its retrospective design. Secondly, as in other similar studies, variability in NLR and PLR during the course of hospitalization was not accounted for. Thirdly, we were unable to collect data on potential confounding factors, such as the use of ACE-inhibitors/angiotensin receptor blockers (ARBs), statins, $\mathrm{N}$-acetyl cysteine, and the administration of isotonic fluid solutions. 
Finally, we did not assess for other causes of acute kidney injury associated with hospitalization, such as the use of diuretics, antibiotics, and other nephrotoxic agents.

\section{Conclusions}

Our study showed a significant correlation between NLR and the incidence of CIN in patients with AMI undergoing cardiac catheterization. NLR is a cheap marker and can be readily calculated at the time of presentation in the emergency department. Our study did not show a significant correlation between PLR and incidence of CIN in AMI patients.

\section{Additional Information \\ Disclosures}

Human subjects: Consent was obtained by all participants in this study. Advent Health Institutional Review Board issued approval 868065. Animal subjects: All authors have confirmed that this study did not involve animal subjects or tissue. Conflicts of interest: In compliance with the ICMJE uniform disclosure form, all authors declare the following: Payment/services info: All authors have declared that no financial support was received from any organization for the submitted work. Financial relationships: All authors have declared that they have no financial relationships at present or within the previous three years with any organizations that might have an interest in the submitted work. Other relationships: All authors have declared that there are no other relationships or activities that could appear to have influenced the submitted work.

\section{References}

1. Wichmann JL, Katzberg RW, Litwin SE, et al.: Contrast-induced nephropathy. Circulation. 2015, 132:193136. 10.1161/CIRCULATIONAHA.115.014672

2. McCullough PA, Adam A, Becker CR, et al.: Epidemiology and prognostic implications of contrast-induced nephropathy. Am J Cardiol. 2006, 98:5K-13K. 10.1016/j.amjcard.2006.01.019

3. Narula A, Mehran R, Weisz G, et al.: Contrast-induced acute kidney injury after primary percutaneous coronary intervention: results from the HORIZONS-AMI substudy. Eur Heart J. 2014, 35:1533-40. 10.1093/eurheartj/ehu063

4. Demircelik MB, Kurtul A, Ocek H, Cakmak M, Ureyen C, Eryonucu B: Association between platelet-tolymphocyte ratio and contrast-induced nephropathy in patients undergoing percutaneous coronary intervention for acute coronary syndrome. Cardiorenal Med. 2015, 5:96-104. 10.1159/000371496

5. Arbel Y, Shacham Y, Ziv-Baran T, et al.: Higher neutrophil/lymphocyte ratio is related to lower ejection fraction and higher long-term all-cause mortality in ST-elevation myocardial infarction patients. Can J Cardiol. 2014, 30:1177-82. 10.1016/j.cjca.2014.05.010

6. Park JJ, Jang HJ, Oh IY, et al.: Prognostic value of neutrophil to lymphocyte ratio in patients presenting with ST-elevation myocardial infarction undergoing primary percutaneous coronary intervention. Am J Cardiol. 2013, 111:636-42. 10.1016/j.amjcard.2012.11.012

7. Kocas C, Yildiz A, Abaci O, et al.: Platelet-to-lymphocyte ratio predicts contrast-induced nephropathy in patients with non-ST-segment elevation acute coronary syndrome. Angiology. 2015, 66:964-68. 10.1177/0003319715578057

8. Mehran R, Aymong ED, Nikolsky E, et al.: A simple risk score for prediction of contrast-induced nephropathy after percutaneous coronary intervention: development and initial validation. J Am Coll Cardiol. 2004, 44:1393-99. 10.1016/j.jacc.2004.06.068

9. The American College of Cardiology's Chest Pain - MI Registry . (2019). Accessed: December 3, 2020: http://cvquality.acc.org/NCDR-Home/registries/hospital-registries/chest-pain-mi-registry .

10. Cho KI, Ann SH, Singh GB, Her AY, Shin ES: Combined usefulness of the platelet-to-lymphocyte ratio and the neutrophil-to-lymphocyte ratio in predicting the long-term adverse events in patients who have undergone percutaneous coronary intervention with a drug-eluting stent. PLoS One. 2015, 10:e0133934. 10.1371/journal.pone.0133934

11. Akcay A, Nguyen Q, Edelstein CL: Mediators of inflammation in acute kidney injury . Mediators Inflamm. 2009, 2009:137072. 10.1155/2009/137072

12. Balta S, Demirkol S, Cakar M, Arslan Z, Unlu M, Celik T: Other inflammatory markers should not be forgetten when assessing the neutrophil-to-lymphocyte ratio. Clin Appl Thromb Hemost. 2013, 19:693-94. 10.1177\%2F1076029613486019

13. Tamhane UU, Aneja S, Montgomery D, Rogers EK, Eagle KA, Gurm HS: Association between admission neutrophil to lymphocyte ratio and outcomes in patients with acute coronary syndrome. Am J Cardiol. 2008, 102:653-57. 10.1016/j.amjcard.2008.05.006

14. Wang X, Zhang G, Jiang X, Zhu H, Lu Z, Xu L: Neutrophil to lymphocyte ratio in relation to risk of all-cause mortality and cardiovascular events among patients undergoing angiography or cardiac revascularization: a meta-analysis of observational studies. Atherosclerosis. 2014, 234:206-13. 10.1016/j.atherosclerosis.2014.03.003

15. Zernecke A, Bot I, Djalali-Talab Y, et al.: Protective role of CXC receptor 4/CXC ligand 12 unveils the importance of neutrophils in atherosclerosis. Circ Res. 2008, 102:209-217. 10.1161/CIRCRESAHA.107.160697

16. Weber C, Zernecke A, Libby P: The multifaceted contributions of leukocyte subsets to atherosclerosis: lessons from mouse models. Nat Rev Immunol. 2008, 8:802-15. 10.1038/nri2415

17. Azab B, Zaher M, Weiserbs KF, et al.: Usefulness of neutrophil to lymphocyte ratio in predicting short- and long-term mortality after non-ST-elevation myocardial infarction. Am J Cardiol. 2010, 106:470-76. 


\section{Cureus}

10.1016/j.amjcard.2010.03.062

18. Park JJ, Jang HJ, Oh IY, et al.: Prognostic value of neutrophil to lymphocyte ratio in patients presenting with ST-elevation myocardial infarction undergoing primary percutaneous coronary intervention. Am J Cardiol. 2013, 111:636-42. 10.1016/j.amjcard.2012.11.012

19. Kurtul A, Yarlioglues M, Duran M, Murat SN: Association of neutrophil-to-lymphocyte ratio with contrastinduced nephropathy in patients with non-ST-elevation acute coronary syndrome treated with percutaneous coronary intervention. Heart Lung Circ. 2016, 25:683-90. 10.1016/j.hlc.2016.01.007

20. Kaya A, Kaya Y, Topçu S, et al.: Neutrophil-to-lymphocyte ratio predicts contrast-induced nephropathy in patients undergoing primary percutaneous coronary intervention. Angiology. 2014, 65:51-56. 10.1177/0003319713484789

21. Yuan Y, Qiu H, Hu X, et al.: Predictive value of inflammatory factors on contrast-induced acute kidney injury in patients who underwent an emergency percutaneous coronary intervention.. Clin Cardiol. 2017, 40:71925. 10.1002/clc.22722

22. Abellás-Sequeiros RA, Raposeiras-Roubín S, Abu-Assi E, et al.: Mehran contrast nephropathy risk score: is it still useful 10 years later?. J Cardiol. 2016, 67:262-67. 10.1016/j.jjcc.2015.05.007

23. Sun XP, Li J, Zhu WW, et al.: Platelet to lymphocyte ratio predicts contrast-induced nephropathy in patients with ST-segment elevation myocardial infarction undergoing primary percutaneous coronary intervention. Angiology. 2018, 69:71-78. 10.1177/0003319717707410

24. Velibey Y, Oz A, Tanik O, et al.: Platelet-to-lymphocyte ratio predicts contrast-induced acute kidney injury in patients with ST-segment elevation myocardial infarction undergoing primary percutaneous coronary intervention. Angiology. 2017, 68:419-27. 10.1177/0003319716660244 